Also available at http://amc-journal.eu ISSN 1855-3966 (printed edn.), ISSN 1855-3974 (electronic edn.)

ARS MATHEMATICA CONTEMPORANEA 8 (2015) 267-273

\title{
Further biembeddings of twofold triple systems
}

\author{
Diane M. Donovan \\ Centre for Discrete Mathematics and Computing, University of Queensland, \\ St Lucia 4072, Australia \\ Terry S. Griggs \\ Department of Mathematics and Statistics, The Open University, \\ Walton Hall, Milton Keynes MK7 6AA, U.K. \\ James G. Lefevre \\ Centre for Discrete Mathematics and Computing, University of Queensland, \\ St Lucia 4072, Australia \\ Thomas A. McCourt \\ Heilbronn Institute, Department of Mathematics, University of Bristol, \\ University Walk, Bristol BS8 1TW, U.K.
}

Received 6 December 2012, accepted 25 January 2014, published online 3 February 2015

\begin{abstract}
We construct face two-colourable triangulations of the graph $2 K_{n}$ in an orientable surface; equivalently biembeddings of two twofold triple systems of order $n$, for all $n \equiv$ 16 or $28(\bmod 48)$. The biembeddings come from index 1 current graphs lifted under a group $\mathbb{Z}_{n / 4} \times \mathbb{K}_{4}$.
\end{abstract}

Keywords: Biembedding, orientable surface, twofold triple system.

Math. Subj. Class.: 05B07, 05C10

\section{Introduction}

A complete graph $K_{n}$ has a triangulation in an orientable surface if and only if $n \equiv$ $0,3,4$ or $7(\bmod 12),[5]$. In such a triangulation the number of faces around each vertex is $n-1$, and so if $n-1$ is even, i.e. $n \equiv 3$ or $7(\bmod 12)$, it may be possible to colour each face using one of two colours, say black or white, so that no two faces of the same

E-mail addresses: dmd@maths.uq.edu.au (Diane M. Donovan), t.s.griggs@open.ac.uk (Terry S. Griggs), jamie.lefevre@gmail.com (James G. Lefevre), tom.a.mccourt@gmail.com (Thomas A. McCourt)

(a) (i) This work is licensed under http://creativecommons.org/licenses/by/3.0/ 
colour are adjacent. In such a case we say that the triangulation is (properly) face twocolourable. In this case the set of faces of each colour class forms a Steiner triple system of $\operatorname{order} n, \operatorname{STS}(n)$ for short, i.e. a collection of triples which have the property that every pair is contained in precisely one triple. We say that the two $\operatorname{STS}(n) \mathrm{s}$ are biembedded in the (orientable) surface. An obvious question therefore is whether, for each $n \equiv 3$ or $7(\bmod 12)$, there is a biembedding of some pair of $\operatorname{STS}(n) \mathrm{s}$ in an orientable surface. The answer is in the affirmative; the case $n \equiv 3(\bmod 12)$ is dealt with in [5] and the case $n \equiv 7(\bmod 12)$ in [6].

In [2] an extension of the above was considered. The necessary condition for the graph $2 K_{n}$, i.e. the graph on $n$ vertices with each pair of vertices joined by two edges, to have a triangular embedding in an orientable surface is $n \equiv 0$ or $4(\bmod 6)$. Such triangulations may be face two-colourable, in which case each colour class forms a twofold triple system of order $n, \operatorname{TTS}(n)$ for short, i.e. a collection of triples which have the property that every pair is contained in precisely two triples. Again we say that the two $\operatorname{TTS}(n) \mathrm{s}$ are biembedded in the (orientable) surface. For such biembeddings to admit a cyclic automorphism it is necessary and sufficient that $n \equiv 4(\bmod 12)$ [2] and a complete solution was provided in that paper. However the method is rather complex. In this paper, for the case $n \equiv 28(\bmod 48)$, we give a much simpler construction based on cyclic biembeddings of Steiner triple systems of order $12 m+7, m \geq 0$. These were found by Youngs [6] and index 1 current graphs corresponding to these solutions are readily accessible. They can be found in [3]. The biembeddings of the TTS $(n) \mathrm{s}$ which we obtain from these biembeddings of Youngs however are not cyclic; they have an automorphism group $\mathbb{Z}_{12 m+7} \times \mathbb{K}_{4}$ where $\mathbb{K}_{4}$ is the Klein 4-group.

Further we extend our method to find new biembeddings of $\operatorname{TTS}(n)$ s for $n \equiv$ $16(\bmod 48)$; these have an automorphism group $\mathbb{Z}_{12 m+4} \times \mathbb{K}_{4}$. Finally iterating this latter process we obtain biembeddings of twofold triple systems of order $4^{s}(12 m+4)$ with an automorphism group $\mathbb{Z}_{12 m+4} \times\left(\mathbb{K}_{4}\right)^{s}, s \geq 1, m \geq 0$.

We will also construct our biembeddings from index 1 current graphs lifted under the appropriate current group $G$ of order $g$. These will satisfy the following properties, which are sufficient to construct a biembedding of a pair of $\operatorname{TTS}(n) \mathrm{s}$ in an orientable surface, [5], [4], [3].

(i) Each vertex has degree 3.

(ii) Each edge is assigned a current from the set $G \backslash\{0\}$ so that each current appears exactly once. Note that a current of $i$ in one direction is equivalent to a current of $-i$ in the opposite direction.

(iii) At each vertex, the sum of the directed currents is the identity (Kirchoff's current law, $K C L)$.

(iv) The directions (clockwise or anticlockwise) assigned to each vertex are such that a complete circuit is formed, that is, one in which every edge of the graph is traversed in each direction exactly once.

(v) The graph is bipartite.

Hence, such a current graph has $2(g-1) / 3$ vertices and $g-1$ edges. We use a Möbius ladder graph with $(g-1) / 3$ "rungs". Set $u:=(g-1) / 3$. The formal definition of such a graph is as follows. The vertex set is $\left\{x_{i}, y_{i} \mid 0 \leq i \leq u-1\right\}$ and the edge set is $\left\{\left\{x_{i}, y_{i}\right\},\left\{x_{i}, x_{i+1}\right\},\left\{y_{i}, y_{i+1}\right\} \mid 0 \leq i \leq u-2\right\} \cup\left\{\left\{x_{u-1}, y_{u-1}\right\},\left\{x_{0}, y_{u-1}\right\}\right.$, 

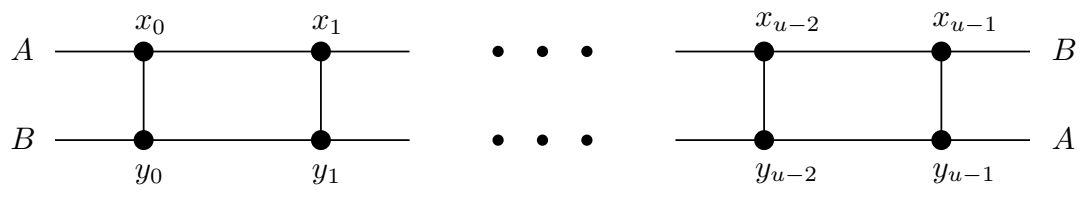

Figure 1: A Möbius ladder graph

$\left.\left\{x_{u-1}, y_{0}\right\}\right\}$. In order to obtain a complete circuit, vertices $x_{i}, 0 \leq i \leq u-1$, and $y_{u-1}$ are assigned a clockwise direction and the vertices $y_{i}, 0 \leq i \leq u-2$, an anticlockwise direction.

In this paper we represent these graphs as shown in Figure 1, where the directions of rotation are not indicated but implicit as defined above.

We build the Möbius ladder graphs with currents assigned to the edges, so that Conditions (ii) and (iii) are met, from gadgets, i.e. edge labelled subgraphs which we link together by concatenation; we will define the linking of two gadgets $D_{1}$ followed by $D_{2}$ by $D_{1}: D_{2}$ and the sequential linking of $k$ gadgets by $\left[D_{i}:\right]_{1 \leq i \leq k}$.

For ease of notation we will label the elements of $\mathbb{K}_{4}$ as $x, y, z$ and 0 , such that

$$
x+x=y+y=z+z=x+y+z=0 .
$$

Hence, the identity element in the group $\mathbb{Z}_{n} \times \mathbb{K}_{4}$ is $(0,0)$.

\section{The case $n \equiv 28(\bmod 48)$}

Let $v=n / 4=12 m+7, m \geq 0$. From [6] there exists a Möbius ladder graph that lifts under $\mathbb{Z}_{v}$ to yield a biembedding of a pair of STS $(v)$ s. Let $\mathcal{L}$ be such a graph.

We begin by labelling Figure 1 as follows, $x_{i}=v_{i}, y_{i}=v_{i+2 m+1}, 0 \leq i \leq 2 \mathrm{~m}$. Thus the bipartition of $\mathcal{L}$ consists of the sets of even-indexed and odd-indexed vertices respectively.

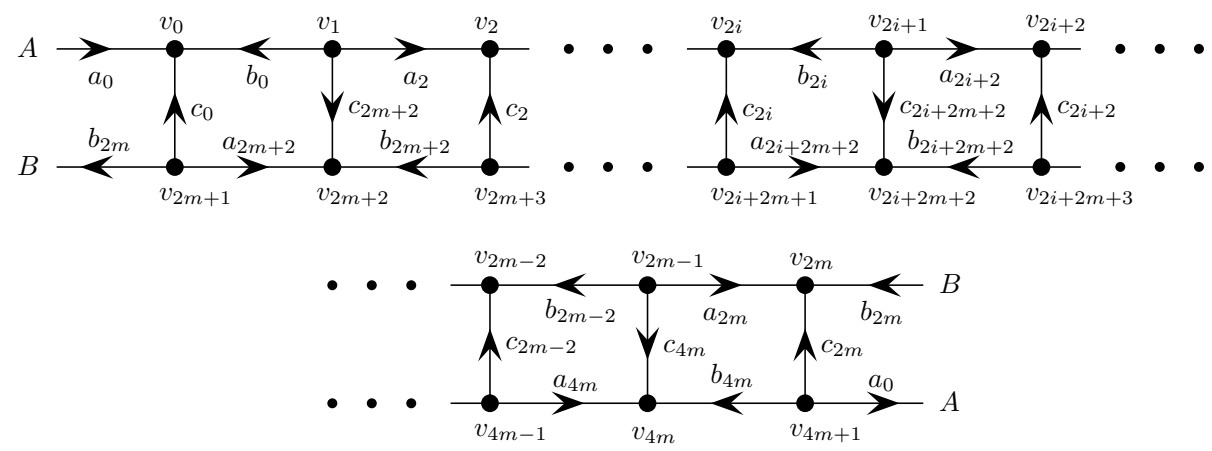

Figure 2: Vertex and edge labels of $\mathcal{L}$. 
Noting that by replacing a directed edge label by its inverse in the opposite direction, we can arrange the directed edge labels so that they point in to even-indexed vertices and out of odd-indexed vertices. Thus the directed edge labels will be taken to be as shown in Figure 2.

As $\mathcal{L}$ satisfies Kirchoff's current law we have the following equations.

$$
\begin{aligned}
a_{i}+b_{i}+c_{i} & \equiv 0(\bmod 12 m+7), \\
a_{i+2}+b_{i}+c_{i+2 m+2} & \equiv 0(\bmod 12 m+7) .
\end{aligned}
$$

We will require the following gadgets where indices are taken modulo $4 m+2$ :

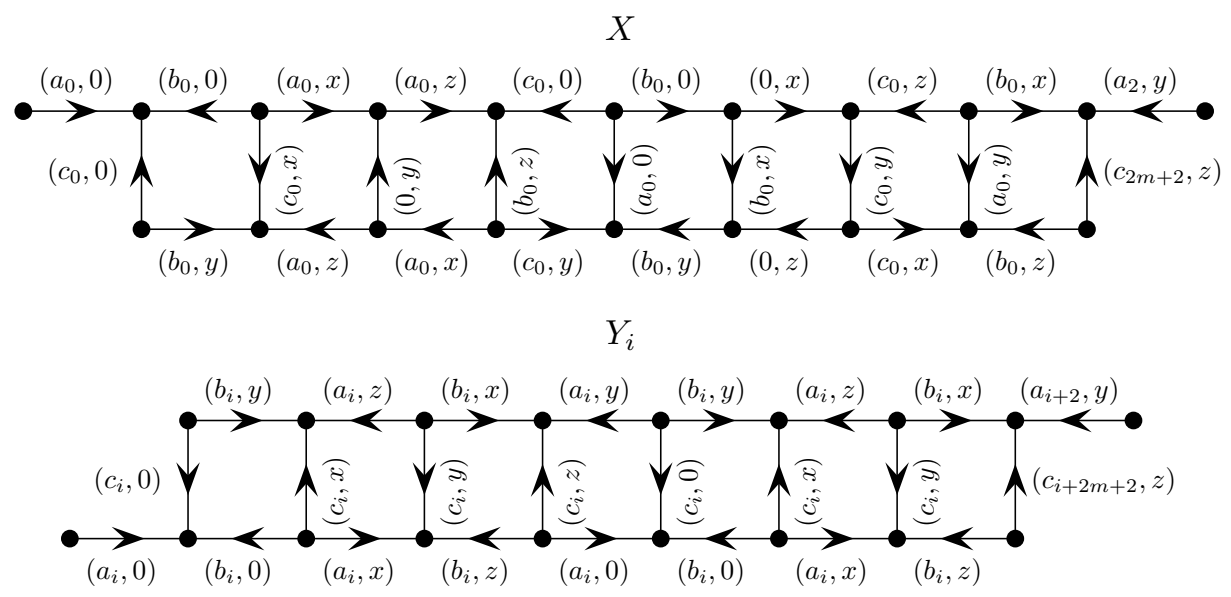

Equations (1.1), (2.1) and (2.2) verify that the gadgets $X$ and, for $1 \leq j \leq 2 m, Y_{2 j}$ satisfy KCL. Now consider the Möbius ladder graph $X:\left[Y_{2 j}:\right]_{1 \leq j \leq 2 m}$. Equations (1.1), (2.1) and (2.2) verify that this graph also satisfies KCL.

Hence, we can lift under the group $\mathbb{Z}_{12 m+7} \times \mathbb{K}_{4}$. Thus, we can construct a biembedding of a pair of TTS $(48 m+28)$ s, $m \geq 0$, with $\mathbb{Z}_{12 m+7} \times \mathbb{K}_{4}$ as an automorphism group.

We conclude this section by giving two examples.

Example 2.1. Let $m=0, v=7$ and $n=28$.

A Möbius ladder graph $\mathcal{L}$, which yields the well known toroidal biembedding of a pair of STS(7)s, has $a_{0}=1, b_{0}=2$ and $c_{0}=-3$, i.e.

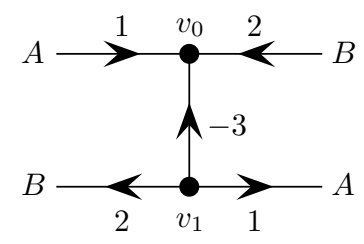

In this case our construction gives just the gadget $X$, labelled as follows.

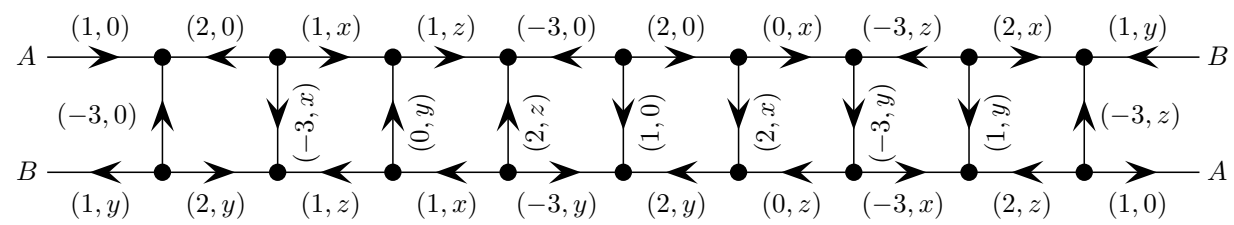


Example 2.2. Let $m=1, v=19$ and $n=76$.

A Möbius ladder graph, $\mathcal{L}$, yielding a biembedding of a pair of STS(19), is as follows.

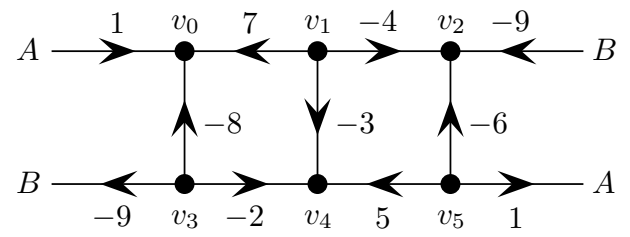

Thus $\left(a_{0}, b_{0}, c_{0}\right)=(1,7,-8),\left(a_{2}, b_{2}, c_{2}\right)=(-4,-9,-6)$ and $\left(a_{4}, b_{4}, c_{4}\right)=$ $(-2,5,-3)$. Our construction gives the following Möbius ladder graph.
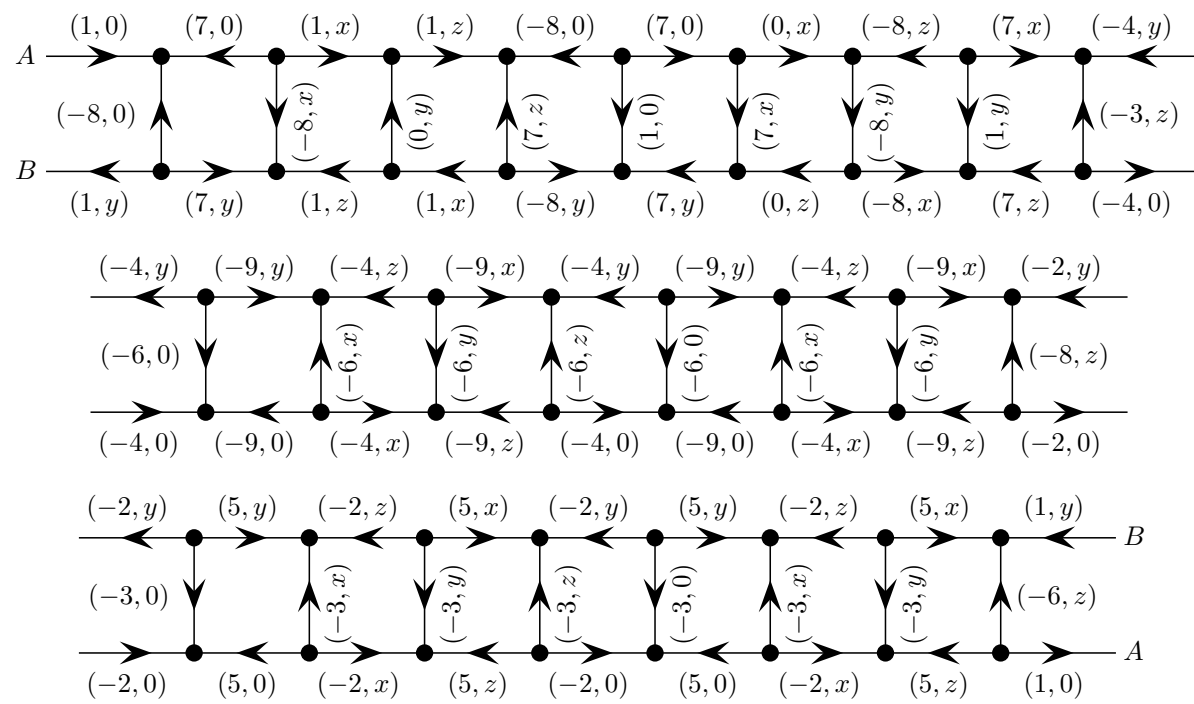

\section{The case $n \equiv 16(\bmod 48)$}

Let $v=n / 4=12 m+4, m \geq 0$. In [2] a Möbius ladder graph $\mathcal{L}$ based on the Colbourn and Colbourn difference triples, [1], on the set $\mathbb{Z}_{12 m+4} \backslash\{0\}$ was constructed. In that paper the ladders were lifted under the cyclic group $\mathbb{Z}_{12 m+4}$ to yield a biembedding of a pair of TTS $(v)$ s. Similarly to Section 2 label the vertices and edges of $\mathcal{L}$ as follows (taking indices modulo $8 m+2$ ):

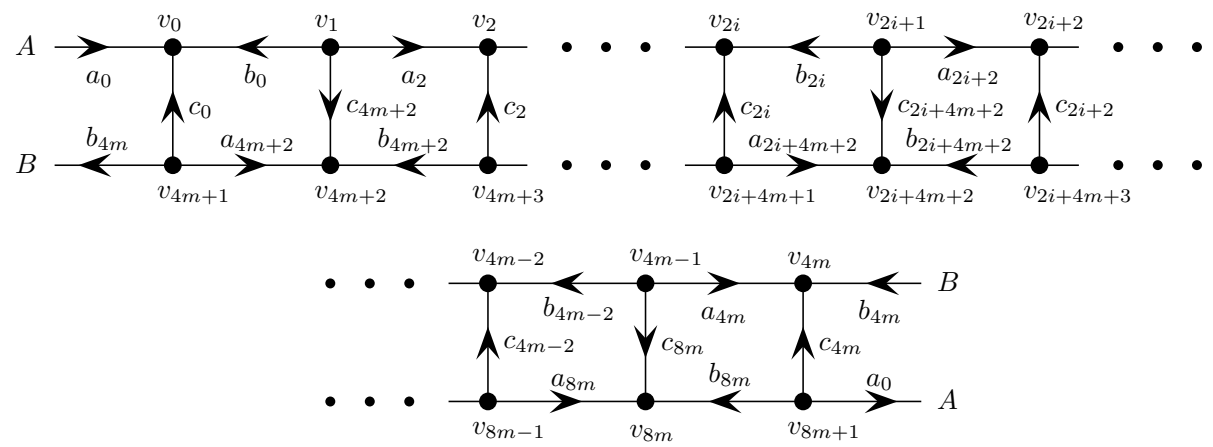


where $v_{0}$ corresponds to the difference triple $3 m+1,3 m+1,6 m+2$. Note that this means that the vertices with even indices correspond to the Colbourn and Colbourn difference triples.

Without loss of generality, in $\mathcal{L}$, either $a_{0}=c_{0}=3 m+1$ and $b_{0}=6 m+2$ or $a_{0}=b_{0}=3 m+1$ and $c_{0}=6 m+2$. Both of these cases occur in the Möbius ladder graphs constructed in [2], depending on the residue class of $v$ modulo 72 and we consider them separately in Subsections 3.1 and 3.2, respectively.

We will make use of the following gadget where $2 \leq i \leq 8 \mathrm{~m}$ :

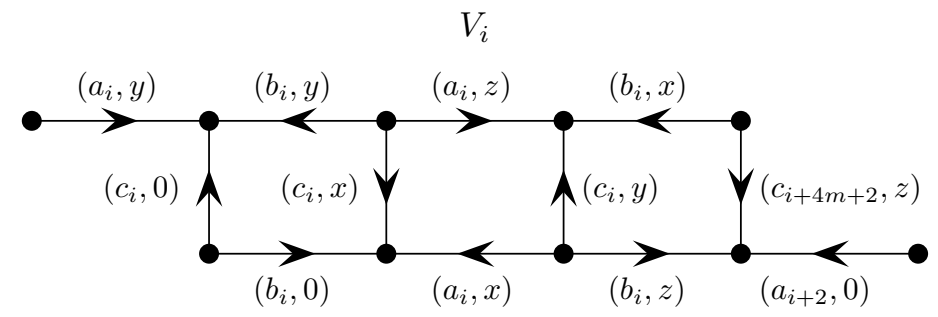

Note that in this case, because the initial Möbius ladder $\mathcal{L}$ yields a biembedding of a pair of twofold triple systems $V_{i}$, is simpler than the gadget used in Section 2. In fact it is "half" that gadget.

As $\mathcal{L}$ satisfies Kirchoff's current law we have the following two equations.

$$
\begin{aligned}
a_{i}+b_{i}+c_{i} & \equiv 0(\bmod 12 m+4), \\
a_{i+2}+b_{i}+c_{i+4 m+2} & \equiv 0(\bmod 12 m+4) .
\end{aligned}
$$

These two equations together with Equation (1.1) verify that $V_{i}$ also satisfies KCL.

\section{$3.1 a_{0}=c_{0}=3 m+1$ and $b_{0}=6 m+2$}

In this case it follows, from [2], that $\mathcal{L}$ yields the following two equations

$$
\begin{aligned}
(6 m+2)+c_{4 m+2}+a_{2} & \equiv 0(\bmod 12 m+4), \\
b_{8 m}+c_{4 m}+(3 m+1) & \equiv 0(\bmod 12 m+4) .
\end{aligned}
$$

In this case we will require the following gadget:

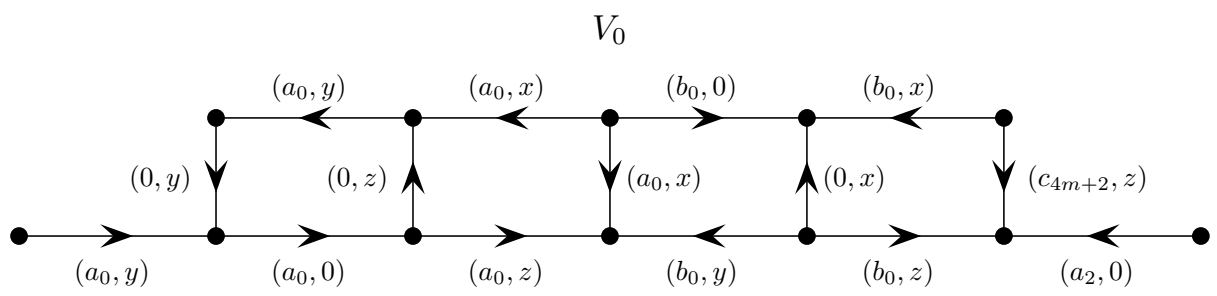

Equations (1.1) and (3.3) verify that $V_{0}$ satisfies KCL.

As $a_{0}=3 m+1$, Equations (1.1), (3.1), (3.2), (3.3) and (3.4) verify that the Möbius ladder graph $V_{0}:\left[V_{i}:\right]_{i=2 j, 1 \leq j \leq 4 m}$ satisfies KCL. 


\section{2 $\mathrm{a}_{0}=\mathrm{b}_{0}=3 \mathrm{~m}+1$ and $\mathrm{c}_{0}=6 \mathrm{~m}+2$}

In this case it follows, from [2], that $\mathcal{L}$ yields the following two equations

$$
\begin{aligned}
(3 m+1)+c_{4 m+2}+a_{2} & \equiv 0(\bmod 12 m+4), \\
b_{8 m}+c_{4 m}+(3 m+1) & \equiv 0(\bmod 12 m+4) .
\end{aligned}
$$

In this case we require the following gadget:

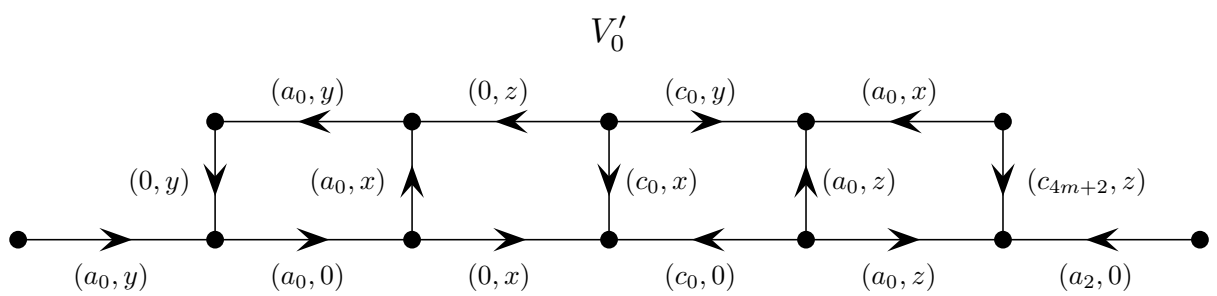

Equations (1.1) and (3.5) verify that $V_{0}^{\prime}$ satisfies KCL.

As $a_{0}=3 m+1$, Equations (1.1), (3.1), (3.2), (3.5) and (3.6) verify that the Möbius ladder graph $V_{0}^{\prime}:\left[V_{i}:\right]_{i=2 j, 1 \leq j \leq 4 m}$ satisfies KCL.

Thus, we have constructed $\left(\overline{\mathbb{Z}}_{12 m+4} \times \mathbb{K}_{4}\right)$-biembeddings of a pair of TTS $(48 m+16)$ s, $m \geq 0$.

Finally note that the gadget $V_{0}$ contains a vertex with currents $(3 m+1, x),(3 m+1, x)$ and $(6 m+2,0)$ pointing outwards. Similarly the gadget $V_{0}^{\prime}$ contains a vertex with currents $(3 m+1, z),(3 m+1, z)$ and $(6 m+2,0)$ also pointing outwards. We call this vertex $\alpha$. Our constructed Möbius ladder graphs $\mathcal{L}^{\prime}$ contain just one of these two gadgets. By reversing the directions on all of the edges of $\mathcal{L}^{\prime}$, labelling the vertex $\alpha$ as $v_{0}$ and the edge with label $(6 m+2,0)$ as $b_{0}$, and extending in the same manner as above, the construction, using the gadget $V_{0}$, can be reapplied. Repeated application of this process yields $\left(\mathbb{Z}_{12 m+4} \times\left(\mathbb{K}_{4}\right)^{s}\right)$ biembeddings of a pair of $\operatorname{TTS}\left(4^{s}(12 m+4)\right) s, s \geq 1, m \geq 0$.

\section{References}

[1] M. J. Colbourn and C. J. Colbourn, Cyclic block designs with block size 3, European J. Combin. 2 (1981), 21-26.

[2] D. M. Donovan, T. S. Griggs, J. G. Lefevre and T. A. McCourt, Cyclic biembeddings of twofold triple systems, Ann. Comb. 18 (2014), 57-74.

[3] M. J. Grannell and T. S. Griggs, Designs and Topology, in Surveys in Combinatorics 2007, A. J. W. Hilton and J. Talbot (Editors), London Math. Soc. Lecture Note Series 346, Cambridge Univ. Press, Cambridge (2007), 121-174.

[4] J. L. Gross and T. W. Tucker, Topological Graph Theory, John Wiley, New York (1987).

[5] G. Ringel, Map Color Theorem, Springer-Verlag, New York (1974).

[6] J. W. T. Youngs, The mystery of the Heawood conjecture, in Graph Theory and its Applications, Academic Press, New York (1970), 17-50. 